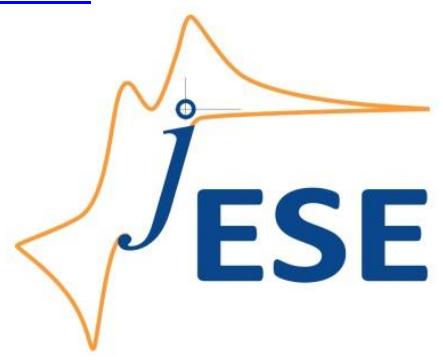

Open Access : : ISSN 1847-9286

www.jESE-online.org

Original scientific paper

\title{
Benchmarking of electrolyte mass transport in next generation lithium batteries
}

\author{
Jonas Lindberg ${ }^{凶}$, Henrik Lundgren, Göran Lindbergh, Mårten Behm
}

Applied Electrochemistry, Department of Chemical Engineering and Technology, School of Chemical Science and Engineering, KTH Royal Institute of Technology, SE-100 44 Stockholm, Sweden

Corresponding authors E-mail: ${ }^{凶}$ ionas13@kth.se; Tel.: +46-8-7908171

Received: July 14, 2017; Revised: August 15, 2017; Accepted: August 15, 2017

\begin{abstract}
Beyond conductivity and viscosity, little is often known about the mass transport properties of next generation lithium battery electrolytes, thus, making performance estimation uncertain when concentration gradients are present, as conductivity only describes performance in the absence of these gradients. This study experimentally measured the diffusion resistivity, originating from voltage loss due to a concentration gradient, together with the ohmic resistivity, obtained from ionic conductivity measurements, hence, evaluating electrolytes both with and without the presence of concentration gradients. Under galvanostatic conditions, the concentration gradients, of all electrolytes examined, developed quickly and the diffusion resistivity rapidly dominated the ohmic resistivity. The electrolytes investigated consisted of lithium salt in: room temperature ionic liquids (RTIL), RTIL mixed organic carbonates, dimethyl sulfoxide (DMSO), and a conventional Li-ion battery electrolyte. At steady state the RTIL electrolytes displayed a diffusion resistivity 20 times greater than the ohmic resistivity. The DMSObased electrolyte showed mass transport properties similar to the conventional Li-ion battery electrolyte. In conclusion, the results presented in this study show that the diffusion polarization must be considered in applications where high energy and power density are desired.
\end{abstract}

\section{Keywords}

Li-ion battery; $\mathrm{Li}_{-} \mathrm{O}_{2}$ battery; Room temperature ionic liquid; Diffusion resistivity; Electrolyte mass transport resistivity 


\section{Introduction}

Lithium-ion batteries are found in nearly all portable electronics, and in technologies still to reach a wider commercial breakthrough such as hybrid, plug-in hybrid, and electric vehicles [1, 2]. In order to reach that breakthrough, batteries that can supply higher power densities are needed.

The maximum power density a battery can deliver is limited by its electrolyte mass transport properties that describe the voltage losses associated with ion transport. These voltage losses (polarizations) cause the battery cell voltage to deviate from its equilibrium, i.e. the observed cell voltage is lower than the theoretical value during discharge and higher at charging, resulting in lowered energy efficiency, energy density, and power density of the battery.

Instantly, when a current is applied to the battery, a polarization directly proportional to the current appears, it follows Ohm's law and is thus referred to as ohmic polarization. In the electrolyte, the ohmic polarization is characterized by the ionic conductivity, $\kappa$, that measures the conductance in an electrolyte when no concentration gradients are present. Gradually, as current passes, concentration gradients develop in the battery due to the diffusion and migration of the ions (convection, being the third way of ion transport, can be neglected in a battery using a porous separator). Migration is the transport of ions in an electric field. It is described by the transport number, $t_{+}$and $t_{-}$- for a cation and anion, respectively, describing the relative amount of current carried by each ion. Since cations and anions will have opposing directions of migration, there will be a resistance to mass transport, as electroneutrality cannot be violated, resulting in a polarization. Diffusion is the transport of species against a concentration gradient. It is characterized by the diffusion coefficient, $\tilde{D}$.

As Li-ion and post Li-ion batteries keep developing there is an increase in the number of electrolytes used. The conventional Li-ion battery electrolyte is typically composed of a Li salt, such as lithium bis(trifluoromethulsulfonyl)imide (Li-TFSI) or lithium hexafluorophosphate (Li-PF6), and an organic carbonate solvent mixture of for example ethylene carbonate (EC), propylene carbonate (PC), or diethyl carbonate (DEC). These electrolytes are thermodynamically unstable against the negative electrodes and rely on their ability to form stable passivating films (solid electrolyte interfaces) in order to be used [3]. Electrolytes based on room temperature ionic liquids (RTIL) have a wide electrochemical stability window making them stable against a larger number of electrodes compared with conventional Li-ion electrolytes [4]. RTILs are also praised for their high conductivity and safety [5]. These properties also make RTIL-based electrolytes possible candidates for lithiumoxygen $\left(\mathrm{Li}^{-\mathrm{O}_{2}}\right.$ ) batteries [6]. More frequently, electrolytes based on dimethyl sulfoxide (DMSO) have been used in $\mathrm{Li}-\mathrm{O}_{2}$ batteries as conventional $\mathrm{Li}$-ion electrolytes have been proven unstable in the presence of $\mathrm{O}_{2}$ [7].

The distribution of the total polarization of a battery during a hybrid pulse power characterization test on a conventional Li-ion battery with an organic carbonate electrolyte has been investigated [8]. It was found that the electrolyte polarization due to concentration gradients and the electrolyte ohmic polarization contributed to $15 \%$ and $28 \%$ of the total polarization, respectively. This means that $43 \%$ of the total polarization was directly attributed to the electrolyte (remaining sources of polarization was attributed to contract resistance, diffusion in solid phase, ohmic resistance in solid phase, and activation overpotential). Furthermore, when enough time had passed for the concentration gradient in the electrolyte to reach a steady state its contribution to the polarization was larger than that of the ohmic polarization [9]. To fully describe the time-dependent mass transport, and its associated polarizations, in a non-dilute and non-ideal electrolyte a model based on concentrated electrolyte theory needs to be set up, and the parameters therein, such as diffusion 
coefficients, transport numbers, and thermodynamic factors need to be determined, in addition to the conductivity. The full set of those mass transport parameters has been determined for some electrolytes [9-14], but since the methods for such comprehensive characterizations are still quite complex and time consuming there is also a need for quick benchmarking methods that yield relevant performance metrics.

Little is known about the mass transport in DMSO-based electrolytes. For RTILs the most common electrolyte performance parameter found in the literature today is conductivity [15], although transport numbers have been determined [16], as well as self-diffusion coefficients obtained by NMR [17]. A problem with using parameters such as the above-mentioned ones for benchmarking is that they are intimately linked to the model that was used to define them. One consequence of this is that it makes it difficult to compare parameters for different types of electrolytes, for example a binary liquid electrolyte, a polymer gel electrolyte and an RTIL. A further problem is that the parameters do not give direct information about the magnitude of electrolyte polarization for a certain current density, i.e. the translation of diffusion coefficients and transport numbers into overpotential is not straightforward. A way of benchmarking electrolytes that meet these requirements was suggested by Nyman et al. [9] by introducing the concept of electrolyte mass transport resistivity (EMTR) ${ }^{1}$

\section{Aim}

The purpose of this study is twofold: (I) to demonstrate the usefulness of electrolyte mass transport resistivity (EMTR) as a method for benchmarking electrolyte mass transport limitations and (II) to highlight the essential lithium battery electrolyte properties by a direct comparison of a set of candidate electrolytes for the next generation of Li batteries. This is done by measuring the EMTR of electrolytes based on RTILs, organic carbonates, a mixture of RTIL and organic carbonates, and DMSO. Little is known about the diffusive mass transport in RTIL and DMSO based electrolytes beyond conductivity, thus, making the use of conductivity as the sole figure of merit potentially misleading. Therefore, the use of a method including concentration gradient polarization is well motivated. For all electrolytes, the time dependence of the electrolyte polarization was also examined.

\section{Theory - electrolyte mass transport resistivity}

Electrolyte mass transport resistivity (EMTR) was initially used as a figure of merit, to quantify polarizations with and without concentration gradients, for interpretation of results from full mass transport characterizations based on concentrated electrolyte theory $[9,13,18]$. It has been experimentally used alongside other techniques to evaluate the influence of flame retardants in Liion battery electrolytes [19].

The definition of the EMTR is shown in Eq. 1, where it is expressed in terms of the parameters in the concentrated electrolyte model [9]. However, it was also suggested that it could be determined experimentally in a relatively simple way, by rewriting Eq. 1 in the form of Eq. 2, where the variables only depend on the experimentally measurable quantities conductivity and diffusion potential at steady state.

$$
-\frac{(\partial \Phi / \partial x)}{i}=\frac{1}{\kappa}+\frac{2 R T}{F^{2} c_{\text {salt }}} \frac{c_{\text {solvent }}}{c_{\text {tot }}} \frac{\left(1-t_{+}^{\text {solvent }}\right)^{2}}{}
$$

\footnotetext{
${ }^{1}$ In Nyman et al. it was called normalized potential gradient.
} 


$$
-\frac{(\partial \Phi / \partial x)_{\text {s.s }}}{i}=\frac{1}{\kappa}+\frac{\Delta \Phi_{\text {diff }, . s .} \epsilon^{\beta}}{F^{2} c_{\text {salt }}} \frac{1}{i}
$$

In these equations $\Phi$ is the electrolyte potential ${ }^{2}, i$ the current density, $k$ the conductivity, $t_{+}$the cationic transport number with respect to the solvent and $\tilde{D}$ the diffusion coefficient with respect to the thermodynamic driving force. $\Delta \Phi_{\text {diff,s.s. }}$ is the potential drop caused by concentration gradients at steady state in a cell of length $I$. The influence of the porosity and tortuosity of the separator is taken into account by multiplying with the porosity, $\epsilon$, raised to the power of the Bruggeman constant $\beta$. The first term on the right hand side of Eq. 2 is the ohmic resistivity, the second term is called the diffusion resistivity ${ }^{3}$. Note that when Eq. 1 is rewritten as Eq. 2 there is no longer a model dependence and Eq. 2 can therefore be used to describe any electrolyte. Further, the method is not limited to dilute and/or binary electrolytes.

The diffusion polarization can be obtained in a relatively simple galvanostatic polarization experiment, and the conductivity is easily obtained by standard methods. By measuring both ohmic and diffusion resistivities the relative contribution of these two sources to the full steady-state electrolyte polarization is obtained. Another way of understanding the EMTR is that multiplied with a given current density it represents the corresponding electrolyte polarization in the electrolyte at steady state.

\section{Experimental}

\section{Materials}

The salt used was Li-bis(trifluoromethanesulfonyl)imide (Li-TFSI, Sigma-Aldrich 99.95 \% purity). The solvents used were dimethyl sulfoxide (DMSO, SigmaAldrich, Anhydrous), N-ethyl-N-methylimidazoleum-TFSI (EMI-TFSI, Solvionic, $99.9 \%$ purity), N-propyl-N-methyl-pyrrolidinium-TFSI (Pyr13-TFSI, Solvionic, $99.9 \%$ purity) and N-propyl-N-methyl-piperidinium-TFSI (Pip13-TFSI, Solvionic, $99.9 \%$ purity). The organic carbonate electrolyte consisted of Li-TFSI salt in ethylene carbonate:diethyl carbonate (EC:DEC) with a 1:1 weight ratio (Novolyte, battery grade). All chemicals were used as received. All RTIL electrolytes contained 1.0 M Li-TFSI, for the DMSO and EC:DEC electrolytes the Li-TFSI content was also varied. The experiments were conducted at $25^{\circ} \mathrm{C}$ in an argon-filled glove box.

\section{Ohmic resistivity experiment}

The conductivities were measured using a Consort K912 conductometer with a SK21T microelectrode probe. The conductometer was calibrated outside of the glove box using potassium chloride standard solutions. The ohmic resistivity was then calculated by using the first term on the right-hand side of eq. 2 .

\section{Diffusion resistivity experiment}

In order to determine the diffusion resistivity a symmetrical test cell with two Li-metal electrodes was assembled. To ensure a constant inter-electrode distance the electrodes were separated by a Teflon spacer-ring with a thickness of $500 \mu \mathrm{m}$ and an inner diameter of $6 \mathrm{~mm}$. The electrolyte was

\footnotetext{
2 The electrolyte potential is defined and measured with a metal reference electrode of the same kind as the cation. The EMTR is therefore also defined for that species.

${ }^{3}$ Note that this term describes both migration and diffusion, it contains both $t_{+}$and $\widetilde{D}$, and both these modes of transport will give rise to concentration gradients. The term "diffusion resistivity" is simply chosen to agree with previously used terminology.
} 
soaked into a Whatman GF/A glass micro fiber filter, placed inside the spacer-ring. The filter has a porosity of 0.9 , and the Bruggeman coefficient has previously been determined to 3.44 [9].

The diffusion potential ( $\Delta \Phi_{\text {diff }}$ in Eq. 2 ) was measured in the test cell directly after a galvanostatic polarization. A schematic of the experimental raw data is seen in Figure 1 . The evolution of the diffusion potential with polarization time could be followed by measuring the potential during current interrupts which were very short in relation to the time scale of the total polarization. Typically, a galvanostatic current was applied for several minutes followed by a two seconds interrupt. When the measured diffusion potential no longer showed any change with polarization time the electrolyte concentration gradients was assumed to be at steady state. The steady state diffusion potential was inserted into the second term on the right-hand side of Equation 2 in order to calculate the diffusion resistivity. The magnitude of the polarization current was chosen so that the potential directly after current switch-off was between 5 and $50 \mathrm{mV}$. The lower potential limit was chosen to give low noise contribution, and the upper limit was set to avoid dendrite formation. Each electrolyte composition was tested in at least four test cells and each cell was polarized with at least four different currents giving potential drops at steady state spanning over 5 to $50 \mathrm{mV}$.

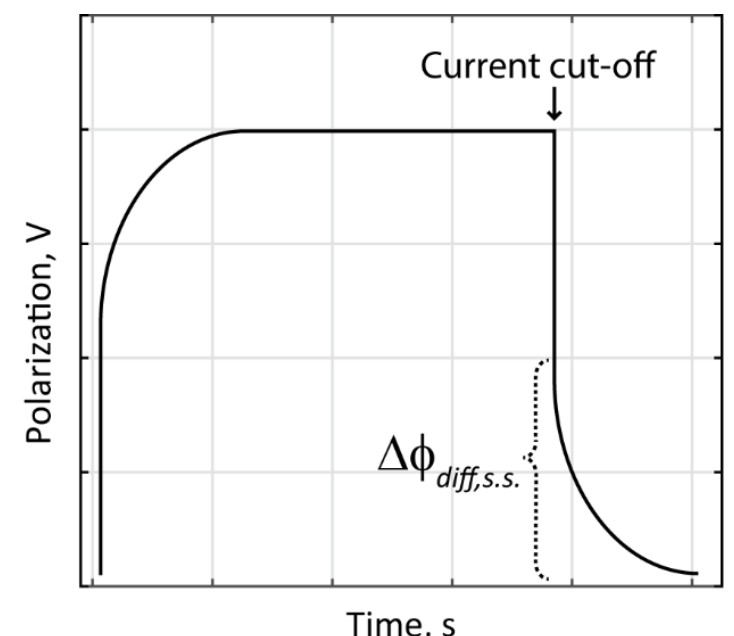

Figure 1. Schematic of the galvanostatic polarization experiment raw data. At the start of the experiment a constant current is applied. When steady state is reached the current is turned off, the potential remaining at this point is caused by the concentration gradients in the electrolyte and corresponds to the diffusion potential,.$\Delta \Phi_{\text {diff }}$ This value is used in Equation 2 in order to calculate the diffusion resistivity

The measurements turned out to be sensitive to the surface of the Li foil, giving less spread in results when pristine Li foil was used. Due to thermodynamic instability between the electrolyte and the Li-metal electrodes, there might be mixed potential between the solvent reduction and the lithium oxidation, thus affecting the measured potential. Since the mixed potential would be solvent dependent its influence would differ from solvent to solvent. However, if the concentration gradients was allowed to fully relax after the galvanostatic polarization the potential difference between the two Li electrodes were in the order of $\mu \mathrm{V}$ for all electrolytes in this study, thus, indicating that the there was no significant influence of a mixed potential in this study.

\section{Results and discussion}

By measuring the diffusion polarization at steady state, $\Delta \Phi_{\text {diff }}$ in a galvanostatic current-interrupt experiment and the conductivity, $\kappa$, using a conductivity meter, the diffusion and ohmic resistivity 
was calculated for each electrolyte according to Equation 2. The calculated resistivities for the electrolytes examined at concentrations of $1 \mathrm{M}$ Li-TFSI are seen in Table 1.

Table 1. The mean ohmic and diffusion resistivity of the various electrolytes tested containing $1 \mathrm{M} \mathrm{Li-TFSI}$. The diffusion resistivities are reported with their standard deviations that show that there was a variation of the measured diffusion resistivity between cells (variation between consecutive experiments in the same cell was smaller)

\begin{tabular}{ccc}
\hline & Ohmic resistivity, $\Omega \mathrm{m}$ & Diffusion resistivity, $\Omega \mathrm{m}$ \\
\hline EC:DEC & 1.5 & $3.0 \pm 0.8$ \\
DMSO & 1.0 & $4.6 \pm 1.6$ \\
EMI-TFSI & 3 & $91 \pm 9$ \\
Pyr13-TFSI & 10 & $220 \pm 10$ \\
Pip13-TFSI & 31 & $590 \pm 50$ \\
\hline
\end{tabular}

All electrolytes show a clear difference in the magnitude between ohmic and diffusion resistivity with diffusion resistivity being larger in all cases. Specifically, in the electrolytes based on RTILs the total polarization was dominated by the diffusion resistivity, whereas for EC:DEC and DMSO both ohmic and diffusion resistivity contributed more equally. For example, comparing EMI-TFSI with EC:DEC the ohmic resistivity was twice as high and the diffusion resistivity was $\approx 30$ times as high for EMI-TFSI, thus stressing the importance of evaluating the diffusion resistivity alongside with ohmic resistivity. Organic solvents and RTILs are miscible; it is therefore possible to create RTILorganic mixed electrolytes that combine the RTIL safety features with the lower viscosity of carbonate solvents. Figure 2 displays the two right-hand side terms of Eq. 2 separately for electrolytes consisting of $1 \mathrm{M} \mathrm{Li-TFSI} \mathrm{with} \mathrm{varying} \mathrm{amount} \mathrm{of} \mathrm{EMI-TFSI} \mathrm{and} \mathrm{EC:DEC} \mathrm{in} \mathrm{the} \mathrm{solvent.}$ Both the ohmic and the diffusion resistivity increased with EMI-TFSI content, with the diffusion resistivity being far greater in magnitude at high EMI-TFSI content.

Electrolytes based on EC:DEC and DMSO showed similar ohmic and diffusion resistivity. This shows that DMSO is a suitable electrolyte for $\mathrm{Li}_{-} \mathrm{O}_{2}$ batteries from a mass transport perspective, however, how suitable DMSO is regarding other factors is still debatable.

Both EC:DEC and DMSO electrolytes were tested at several Li-TFSI concentrations, see Figure 3. The lowest EMTR was observed at concentrations above $0.5 \mathrm{M} \mathrm{Li-TFSI}$. Lower concentrations resulted in higher ohmic and diffusion resistivities. The spread in measurements was also larger at lower concentration. A resistivity vs. concentration graph was produced by Nyman et al. from a full characterization of $\mathrm{LiPF}_{6}$ in 3:7 EC:EMC [9] displaying similar results, although the diffusion resistivity is higher in the present study, which might be due to a larger anion inhibiting mass transport. This confirms that the method applied here provides results equivalent to those obtained from complex full characterizations based on physical models.

A reason that the RTILs showed large EMTR may be found by looking at the constituents of the RTIL electrolytes. In the RTILs in this study there are three ionic species present: $\mathrm{Li}^{+}$, TFSI and a bulky cation (EMI, Pyr13 or Pip13). For $1 \mathrm{M} \mathrm{Li-TFSI} \mathrm{in} \mathrm{EMI-TFSI} \mathrm{the} \mathrm{coordination} \mathrm{number} \mathrm{is} \mathrm{a} \mathrm{little} \mathrm{bit} \mathrm{lower}$ than two [20] suggesting that the predominant coordination complex is [Li(TFSI) $\left.{ }_{2}\right]^{-}$.

This would change the effective charge of the current-carrying specie from positive to negative i.e. changing the migration direction of $\mathrm{Li}$, thus making diffusion the only means of transport carrying Li to the cathode. As the RTIL content increases in a mixed electrolyte, so does the viscosity [5]. 
a)

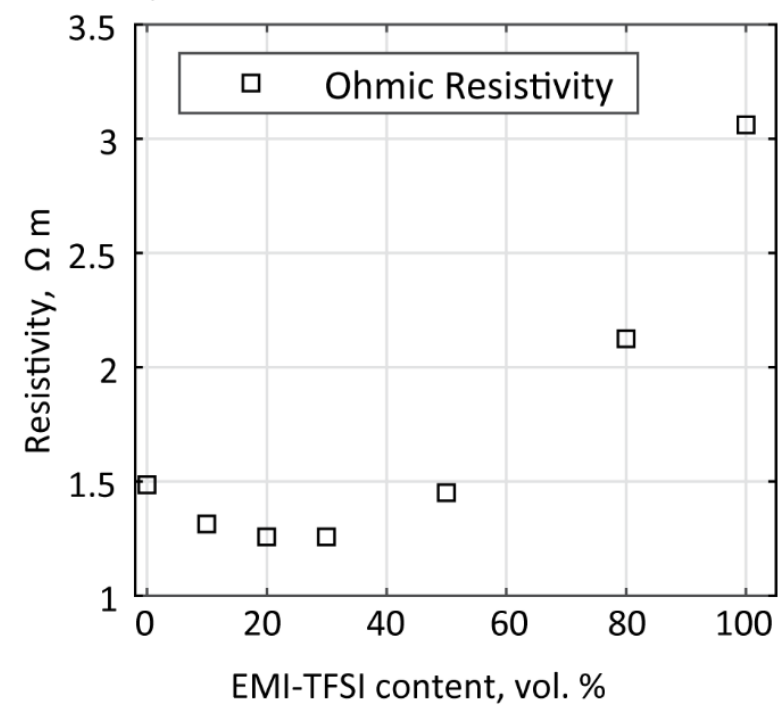

b)

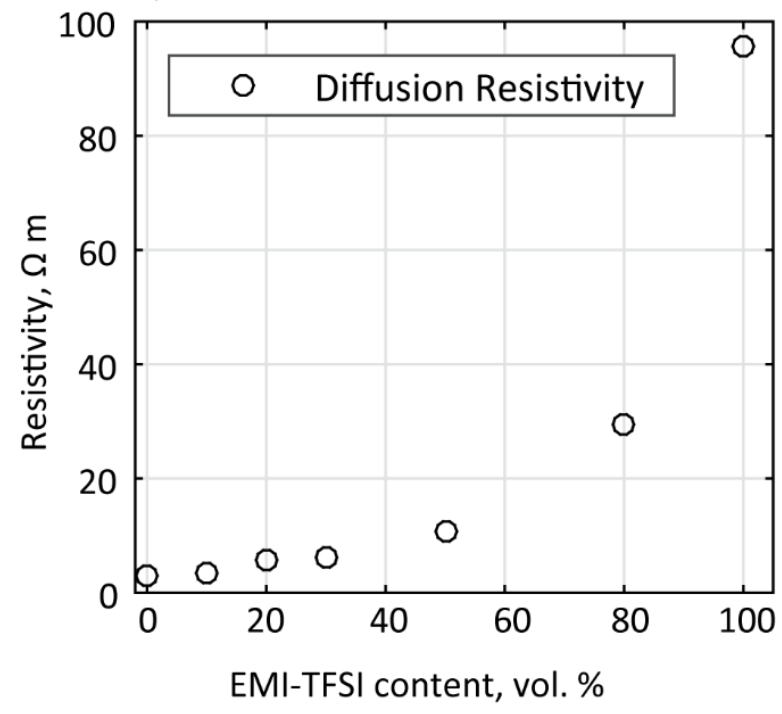

Figure 2. The effect on ohmic ( $\square$ ) and diffusion ( $(\circ)$ resistivity when EMI-TFSI was added to EC:DEC are shown in (a) and (b), respectively. All electrolytes contained 1 M Li-TFSI

a)

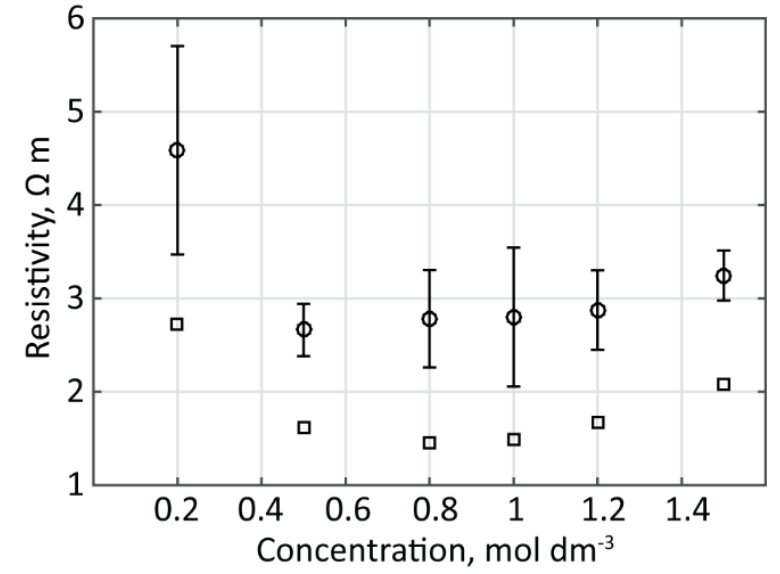

b)

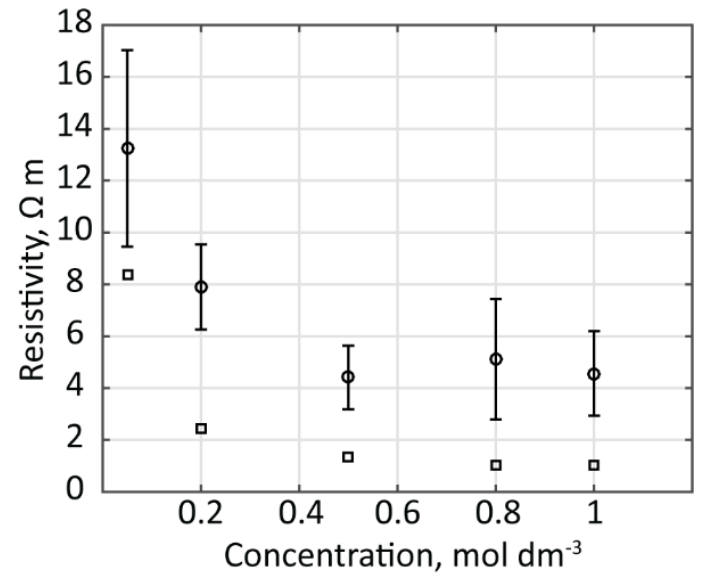

Figure 3. The diffusion ( $(\circ)$ and ohmic $(\square)$ resistivity at different LiTFSI concentrations in (a) EC:DEC and (b) DMSO measured at steady-state. The error bars show standard deviations

However, explaining the variation in mass transport properties in terms of change in viscosity is not suitable since viscosity should affect the ohmic and diffusion resistivities equally and the results do not show this (see Figure 2). An alternative explanation could be found if looking at the system in a molecular perspective. When DEC is the only solvent present the Li ion coordinates to DEC in a $\left[\mathrm{Li}(\mathrm{DEC})_{3}\right]^{+}$complex [21]. In a mixed EC:EMI-TFSI solvent the predominant solvation complex of $\mathrm{Li}^{+}$is $\left[\mathrm{Li}(\mathrm{EC})_{4}\right]^{+}$down to a Li:EC molar ratio of 1:4 $[22,23]$. At an EMI-TFSI content higher than 50 vol.\% in EC:DEC there is not enough EC and DEC to fully solvate all Li ions. When the EMI-TFSI content is increased there is a steep rise in diffusion resistivity suggesting that the solvation of the Li ions is of key importance, see Figure 2. RTILs have promising safety features and addition of RTILs to organic solvents increases safety. An addition of $40-60$ vol.\% EMI-TFSI to $1 \mathrm{M} \mathrm{LiPF}_{6}$ in EC:DEC has been suggested as an optimal composition with no flammability, high conductivity and low viscosity [5]. However, this study shows that at 50 vol.\% EMI-TFSI there is a fourfold increase in diffusion resistivity compared to when no RTIL was present.

The time needed for the diffusion polarization to reach a steady state depends on the interelectrode distance, electrolyte, and the polarization current. In the galvanostatic polarization 
experiment, currents varying by one order of magnitude was needed in order to get diffusion polarizations within the desired range of 5 to $50 \mathrm{mV}$. Therefore, the time needed to reach steady state also varied by one order of magnitude with larger currents needing more time. The diffusion polarization for the electrolytes based on EC:DEC and DMSO typically reached steady state within 10 to 20 minutes for polarizations of $10 \mathrm{mV}$, see Figure 4a. For the RTILs this time was typically 4 to 20 hours, see Figure $4 \mathrm{~b}$. Keep in mind that the test cell had an electrode distance of $500 \mu \mathrm{m}$ and that if the electrode distance were to be reduced one order of magnitude, to better agree with a real battery, then the time would be reduced by two orders of magnitude (according to Fick's second law). This would correspond to 2-12 min for the electrolytes based on the RTILs and 6-12 seconds for those based on EC:DEC and DMSO. Further, the time needed for the diffusion resistivity to equal the ohmic resistivity would be typically five seconds for the RTIL based electrolytes and 1-2 seconds for the electrolytes based on EC:DEC and DMSO. This indicates that losses due to concentration gradients in a Li battery must be considered in most usage scenarios where energy efficiency and power density is important.

a)

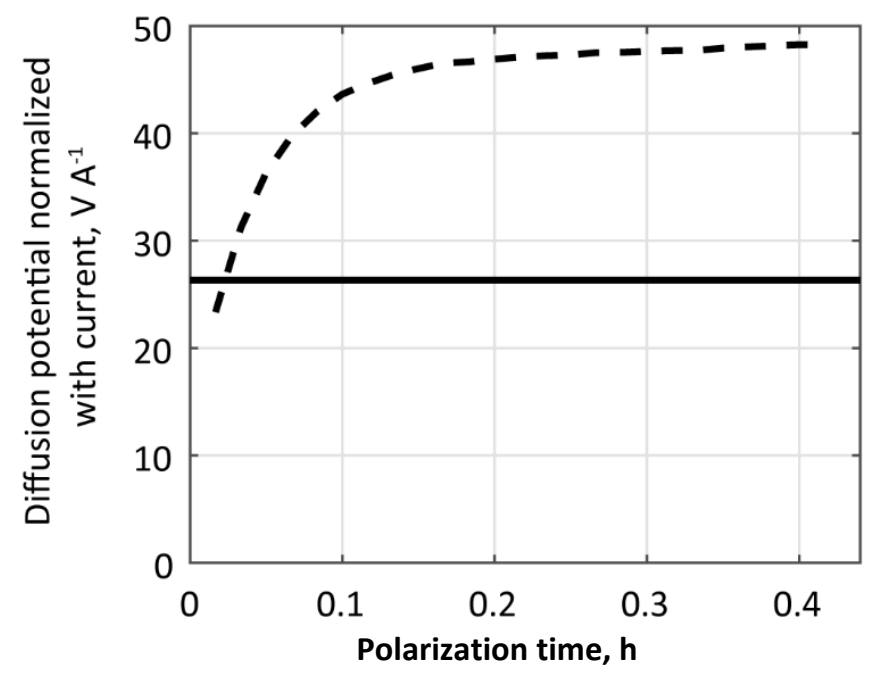

b)

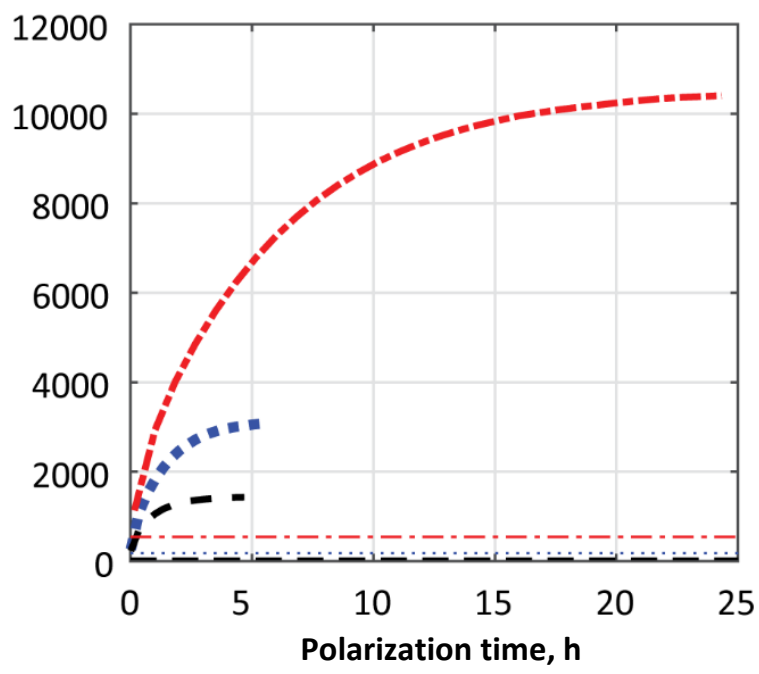

Figure 4. (a) The diffusion potential (-) for 1 M LiTFSI in EC:DEC shown as a function of time. The current normalized ohmic potential is shown as a time-independent horizontal line.

(b) The current-normalized diffusion potentials of EMI-TFSI (-, black), Pyr13-TFSI (-, blue) and Pip13-TFSI (--, red). The current-normalized ohmic potentials are shown as time-independent horizontal lines, EMI-TFSI (-, black), Pyr13-TFSI (', blue) and Pip13-TFSI (--, red). All electrolytes contained 1 M Li-TFSI

\section{Conclusions}

In this study, both ohmic and diffusion voltage losses, presented as resistivities, were measured in a quick and convenient way to examine the mass transport in next generation Li-battery electrolytes. The results highlight the importance of quantifying the diffusion together with the ohmic polarization when trying to estimate the total electrolyte polarization.

When a conventional Li-ion battery electrolyte was examined the results showed good agreement with ohmic and diffusion resistivities from a full characterization based on a physical model, thus, validating the method used in this study. Electrolytes based on dimethyl sulfoxide (DMSO) showed mass transport resistivities similar to those of the conventional Li-ion electrolyte, making the DMSO based electrolytes suitable for use in $\mathrm{Li}_{-} \mathrm{O}_{2}$ batteries from a mass transport perspective. When examining electrolytes based on room temperature ionic liquids (RTIL) the mass transport resistivities were $25-130$. The diffusion resistivity at steady-state was $\sim 20$ times greater 
than the ohmic resistivity for $1 \mathrm{M} \mathrm{Li-TFSI} \mathrm{in} \mathrm{the} \mathrm{RTILs} \mathrm{tested.} \mathrm{This} \mathrm{ratio} \mathrm{was} \mathrm{only} \mathrm{about} \mathrm{two} \mathrm{for}$ conventional Li-ion battery electrolytes. For all the electrolytes examined, the concentration gradients developed quickly enough for the diffusion resistivity to become larger than the ohmic resistivity in short time. When a mixture of RTIL and organic carbonates was used as solvent the diffusion resistivity was larger than when pure organic carbonates were used. Adding more than 50 vol.\% RTIL drastically increased the diffusion resistivity.

As concluding remarks, it can be said that the method employed here, measuring both the ohmic and diffusion resistivity, offers insight to the mass transport of a group of compounds where previously little has been known beyond conductivity and viscosity.

Acknowledgements: Financial support from The Swedish Foundation for Strategic Research (SSF) and The Swedish Electromobility Centre is gratefully acknowledged.

\section{References}

[1] E. J. Cairns, P. Albertus, Annual Review of Chemical and Biomolecular Engineering 1 (2010) 299-320

[2] I. A. Fulton, Renewable and Sustainable Energy Reviews 68 (2017) 685-692

[3] P. Verma, P. Maire, P. Novák, Electrochimica Acta 55 (2010) 6332-6341

[4] M. Galinski, A. Lewandowski, I. Stepniak, Electrochimica Acta 51 (2006) 5567-5580

[5] A. Guerfi, M. Dontigny, P. Charest, M. Petitclerc, M. Lagace, A. Vijh, K. Zaghib, Journal of Power Sources 195 (2010) 845-852

[6] C. J. Allen, S Mukerjee, E. J. Plichta, M. A. Hendrickson, K. M. Abraham, Journal of Physical Chemistry Letters 2 (2011) 2420-2424

[7] S. A. Freunberger, Y. Chen, Z. Peng, J. M. Gri, L. J. Hardwick, F. Barde, P. Novak, P. G. Bruce, Journal of the American Chemical Society 133 (2011) 8040-8047

[8] A. Nyman, T. G. Zavalis, R. Elger, M. Behm, G. Lindbergh, Journal of the Electrochemical Society 157 (2010) A1236-A1246

[9] A. Nyman, M. Behm, G. Lindbergh, Electrochimica Acta 53 (2008) 6356-6365

[10] L. O. Valoen, J. N. Reimers, Journal of the Electrochemical Society 152 (2005) A882-A891

[11] Y. P. Ma, M. Doyle, T. F. Fuller, M. M. Doeff, L. C. Dejonghe, J. Newman, Journal of the Electrochemical Society 142 (1995) 1859-1868

[12] P. Georen, G. Lindbergh, Electrochimica Acta 47 (2001) 577-587

[13] H. Lundgren, J. Scheers, M. Behm, G. Lindbergh, Journal of The Electrochemical Society 162 (2015) A1334-A1340

[14] A. Ehrl, J. Landesfeind, W. A. Wall, H. A. Gasteiger, Journal of The Electrochemical Society 154 (2017) A826-A836

[15] B. Garcia, S. Lavallee, G. Perron, C. Michot, M. Armand, Electrochimica Acta 49 (2004) 4583-4588

[16] J. K. Kim, L. Niedzicki, J. Scheers, C. R. Shin, D. H. Lim, W. Wieczorek, P. Johansson, J. H. Ahn, A. Matic, P. Jacobsson, Journal of Power Sources 224 (2013) 93-98

[17] A. Noda, K. Hayamizu, M. Watanabe, Journal of Physical Chemistry B 105 (2001) 4603-4610

[18] H. Lundgren, M. Behm, G. Lindbergh, Journal of The Electrochemical Society 162 (2015) A413-A420

[19] K. Ciosek Hogstrom, H. Lundgren, S. Wilken, T. G. Zavalis, M. Behm, K. Edstrom, P. Jacobsson, P. Johansson, G. Lindbergh, Journal of Power Sources 256 (2014) 430-439

[20] J. C. Lassegues, J. Grondin, C. Aupetit, P. Johansson, Journal of Physical Chemistry A 113 (2009) 305-314

[21] M. Datt Bhatt, M. Cho, K. Cho, Canadian Journal of Chemistry-Revue Canadienne De Chimie 89 (2011) 1525-1532

[22] J. C. Lassegues, J. Grondin, D. Talaga, Physical Chemistry Chemical Physics 8 (2006) 5629-5632

[23] L. J. Hardwick, M. Holzapfel, A. Wokaun, P. Novak, Journal of Raman Spectroscopy 38 (2007) 110-112

C2017 by the authors; licensee IAPC, Zagreb, Croatia. This article is an open-access article distributed under the terms and conditions of the Creative Commons Attribution license

(http://creativecommons.org/licenses/by/4.0/) 